\title{
DESIGN FACTORS FOR A SUCCESSFUL SHARED SPACE STREET (SSS) DESIGN
}

\author{
Ranmalsingha R. J. C. JAYAKODY, ${ }^{1,}$, Kaushal KERAMINIYAGE ${ }^{1}$, \\ Mark ALSTON ${ }^{2}$, Nuwan DIAS ${ }^{1}$ \\ ${ }^{1}$ School of Art, Design and Architecture, University of Huddersfield, Huddersfield, UK \\ ${ }^{2}$ School of the Built Environment, University of Salford, Manchester, UK
}

Received 2 December 2015; accepted 6 April 2017

\begin{abstract}
The concept of Shared Space Street (SSS) has the potential to bring many benefits to a city. Those include promotion of social interaction, the connectivity within the city for both vehicles and pedestrians, active engagement of the people with the space, walkability, vitality and street livability, better economic wealth and alike. These factors work together to improve livability, vitality of street and indirectly bring economic wealth to municipalities through increasing the footfall to shops, enhancing the health and safety of the locality and increasing the property values. Hence, this clearly is a consideration for strategic property management and relevant professionals. This concept has also been criticized for its practical issues when implemented in some parts of the world. Such issues include difficulties faced by aged people and people with disabilities, harassments faced by the cyclists, etc. This paper explores the methods and approaches that can be used to harness potential advantages of the SSS concept and to overcome its practical issues and criticisms through a detail evaluation of design driven use of space in three case studies within United Kingdom. Finally, this paper proposes a set of design factors which can be applied to a SSS design in order to ensure a successful implementation.
\end{abstract}

Keywords: Shared Space Street, pedestrian prominence, inclusive design, distinctive and attractive streets, connectivity.

\section{Introduction}

The concept of Shared Space Street (SSS) promotes the notion of sharing a single space by both pedestrians and vehicles while the prominence is given for pedestrian movement. This concept was first emerged in Europe and North America and further developed in Denmark, Northern Holland, Sweden and Northern Spain (Hamilton-Baillie, 2008). Currently, this concept is widening as a contemporary method of creating walkable, well connected, pedestrian prominent streets in modern cities. The basic notion of SSS has been noted in the literature as, sharing a single space by pedestrian, cyclist and vehicles, while promoting the freedom of movement for pedestrians and ensuring the connectivity of vehicular and cycle movement (Gehl, 2013; Department for Transport, 2011; Carmona, Tiesdell, Heath, \& Oc, 2003; Kaparias et al., 2013).

In the history of transportation, streets were shared by horse carriages, bullock carts, pedestrians and cars without any traffic management measures. Then with the increase of the vehicular users, streets became unsafe for pedestrians, and as a solution, sidewalks were introduced. Subse- quently, in the $20^{\text {th }}$ century, due to the growing number of vehicles, a major separation of vehicular traffic from civic was placed with the introduction of pedestrian bridges and underpasses. That separation resulted in segregated movement networks for pedestrians and vehicles. Further, for the last sixty years when designing streets, the priority was given for the drivers, the "well-designed" streets means ease of drive and less traffic (CABE, 2008). As such, the needs of the community such as walking, sitting and relaxing, shopping, playing, and cycling were treated as low priority. Understandably, these design practices received criticisms from those who respected the social value of urban streets. However, the vehicular traffic can discourage the pedestrians, and disrupt the social values of urban streets, yet the spatial segregation can hardly be a solution as it decreases the connectivity and can result inconvenience for all the consumers (Carmona et al., 2003). Furthermore, this segregation or prioritisation of vehicular movement, leads to decrease the quality of streets by removing the characteristics such as community vitality, diversity, distinctiveness and urban quality. This follows

*Corresponding author. E-mail: Chathuranganee.Jayakody@hud.ac.uk 
the view of researchers such as Jacobs (1995), where he states that the streets and intersections which welcome all modes of transportation are the places where pedestrians and drivers work best, distinctive, friendly, and the places that help to build the local community.

Within this context, the concept of "Shared Space Street (SSS)" was emerged with a view of protecting social value of spaces while ensuring the connectivity of vehicular network. Further, most of the urban design concepts related to sustainable urban development confirmed the value of this concept as a mode to create sustainable cities. Especially, the New Urbanism which is a radical urban design movement emerged in early 1980s, agrees that the shared spaces can be used as a mode to promote the elements such as the sense of community and neighbourhood form (Talen, 1999), through facilitating the walkability and well-connected streets with pedestrian comfort and to promote the local distinctiveness and diversity (Hebbert, 2003). Indeed, most of the older people find their own freedom through walking in to livable street, may be to post a letter, to buy a newspaper, walk with the dog or just to get some fresh air (Burton \& Mitchell, 2006).

SSS facilitates the creation of livable places through the encouragement of walking and engagement with the space hence, indirectly contributes to social security (Babbage Science and Technology, 2012). Further, SSS encourages the walking and active engagement of public (Curl, Thompson, \& Aspinall, 2015) which indirectly contributes to the health and wellbeing of the community. Apart from that, CABE (2008) research found that SSS can create more economic wealth to the area as it acts as an attractive public realm, increases the foot falls for local shops and increases the value of real estate. Hence this has a direct impact on the surrounding properties, which needs careful management. Indeed, SSS has the potential to bring many benefits to cities in multiple ways contributing positively to social, economic and environment.

Nevertheless, the concept of SSS has also been criticised mainly for its operational issues. The major criticism is that the aged people or people with hearing difficulties and blind or partially blind people do not feel safe to be in this type of street as they cannot see or hear the vehicular movements, especially cycle movements (Carmona et al., 2003). Another criticism is that the SSS design cause issues to cyclists, especially when cycles and vehicles move in one direction, as cyclist may be intimidated by cars passing too close. Moreover, cyclists need to be much more careful about pedestrians when they all occupy the same space (MacMichael, 2009). The third criticism is more subjective or attitudinal which describes user's dislike for the streets without more familiar road elements such as kerbs, crossings, signage, etc. Therefore, in some instances, these SSSs are sometimes called "Naked Streets" (MacMichael, 2009).

A carefully designed space, however, can overcome most of these criticisms if those issues are treated appropriately within the design process. Often, the unpredictability of design outcomes and unfamiliarity with the concept, contribute to most of these issues (CABE, 2008). Accordingly, this research paper investigates how to design a SSS harnessing its potentials and overcoming the constraints and criticisms. This warrants an in-depth analysis of design factors which need to be considered for the successful implication of SSS concept.

\section{Literature review}

The aim of this study is to investigate and establish the factors which contribute to the design of successful SSS'. Within this scope, it was important to establish the success factors for SSS. In other words, in the first instance, A comprehensive literature review was conducted to identify these success factors, through a critical evaluation of journal papers, book chapters, conference papers, newspaper articles, as well as local and international institutional reports which discuss the potentials and issues in the subject area. Accordingly, four main principles were identified as the measures of effectiveness of Shared Space Streets.

\subsection{Pedestrain prominence}

Gehl (2013), state that mixing the traffic group is not merely a shared space until a clear priority is given for the pedestrian. Department for Transport (2011) reveal six main design elements which are focused on creating freedom of pedestrian movement. Those are; Low vehicle speed with visual narrowing; Application of courtesy crossings; Design of transitional zone; Level of surface with no level differentiation to divide the carriage way, pedestrian way and cycle way; Comfort spaces; and A space for parking and loading activities should be allocated without interrupting pedestrian movement and activities. Further, it has been identified that the allocation of spaces for cycle parking is an important factor to discourage the informal cycle parking which can be inconvenient for pedestrian activities (Department for Transport, 2011).

Furthermore, case examples such as New Road, Brighton, United Kingdom (Healthy cities, 2012), Kelheim Bavaria, Germany (Pharoah, 2008) and High Street Rijksstraatweg in Haren, Netherlands (Gerlach, Methorst, Boenke, \& Leven, 2008), provide evidence on how the focus on pedestrian prominence contribute to successfulness of SSS design. Some of these streets were vehicular dominant and unattractive, in some cases streets were with fear of anti-social behaviour and unwelcoming for visitors. These streets were transformed into SSS with increased number of pedestrians, lower speed of traffic, less conflicts between vehicle users, pedestrians and cyclists, and users spend more time in the street. Theses case examples support the notion that design for pedestrian friendly environment contributes to the success of SSS together with the design features such as low speed of traffic by the design, activities, existence of pedestrian and minimal physical visual segregation of space. Further, these examples establish that the removal of traffic rules such as signals, signs, divisions, median lines, markings can contribute to create the pedestrian friendly environment, 
with the proviso that this removal should not be done just because it is a shared space, but with a proper justification and with a clear intension of achieving the objectives of a shared space. Further, the studies of Curl et al. (2015) provide evidences on how all these factors act together to improve the pedestrian perception of how easy it is to walk on the street.

Public Realm Information \& Advice Network (2011) emphasises the need for strong entrance designs with reduced width of carriageway which inform the drivers that they are entering a shared space and not a conventional street. In supporting this view, the importance of entrance design was confirmed by Hamilton-Baillie (2007), through their study on few examples of SSS across the world. Furthermore, they state that the entrance designs act as a transition point with reduced width of the carriage way thus slowing traffic speed.

\subsection{Distinctive and attractive public place}

Second main feature is related to "place making" which covers the elements such as active engagement of public with the space, encourage diversity, social interaction and distinctive and attractive place for people. Pharoah (2008), studied a shared street case in Kelheim which is a historic town Centre in Germany. He points out that the design of shared space as a place for people, place for shop, place to relax, place to visit, can be considered as one of the main distinct features of the SSS. Further, Healthy Cities (2012) confirms that most of the SSS users were attracted to street features such as seating, restaurants/bars, self-congestion, lighting and atmosphere. Adding to this, Karndacharuk, Wilson, and Dunn (2014), highlight that the designed stone pavers, along with a suite of street furniture (e.g. seats, cycle racks, lighting and native trees) can be used in achieving the goal of distinct area with a sense of place. The above promotes the social value of urban streets which is rooted to Jane Jacob's school of thought, "if a street of a city looks interesting, city looks interesting, if streets look dull, city looks dull" (Jacobs, 1995). Indeed, as the above denotes, successful shared street have the potential to add distinctive and attractive place to the city.

In a similar vein, Hamilton-Baillie (2007), describes that the design of shared spaces as an attractive place for the people with the sense of the place. Indeed, this is one of the main reasons driving the successful application of SSS concept. To highlight his views about shared spaces, Hamilton-Baillie (2007) presents a discussion on five popular SSS examples: London - Kensington High Street, Devizes - Market Square, UK, The High Street of Lyngby in the northern suburbs of Copenhagen, The High Street (Rijksstraatweg) in Haren, near Groningen, Netherland and Svallertorget (Gossip Square), Norrköping, Sweden. These case examples display how SSS can create attractive places with sense of place, through the improvement of relationship between the building, street and activities. Further, they showcase that this improvement can be done through the landscaping, paving, lightning, active building frontage, public art and through the consideration on making public realm by using special events, shop displays, street markets and alike.

In order to create distinctive and attractive public place, literature findings suggest the need of having comfort places. "Comfort place" denotes an area allocated to street users to rest or to engage with different activities without any interference from vehicles and cycles. Mostly, these comfort places provide space for seating and engagement in other activities (Department for Transport, 2011). Further these comfort places provide space for seating, ease of pedestrian movement, active engagement of activities and alike. Also, provision of plenty of open-air private and public seating is one of the main redesigning attributes of shared space (Healthy Cities, 2012).

\subsection{Inclusive design}

One of the main criticisms towards SSS is that this street excludes the blind and partially sighted people, people with moving disabilities and aged people. Especially, for people who cannot see or hear the vehicular movement, feel unsafe to be on this type of street. With these arguments Imrie (2012), state that Shared space can be understood as a manifestation of disabling design in the built environment, and as a reaffirmation of disabled people's inabilities. For instance, shared space at Hales Street, UK came under scrutiny after a pensioner was hit by a bus on January 2012 (Matthew, 2012). Within this context, The Guide Dogs for the Blind Association (2014) highlights that, for blind and partially sighted people, it is dangerous to bring the road and the pedestrian path (pavement) to the same level in a bid to make it a "shared space" as they rely upon the presence of the kerb to know that they are on the pavement and not on the road.

Adding to this, Holmes (2015), states that shared surface (road and the pedestrian path at the same level), effectively excludes the people with disabilities from the street environment and guide dogs for the blind cannot find the way, especially when crossing the street. As the above highlights, if the space is designed without considering all the groups of the society, such places create issues and exclude the people with special needs from the environment.

The above criticisms elevate the need of looking for design solutions to mitigate the issues related to Shared Space Streets. At the outset the question will then be, is it possible to plan and design the Shared Space Streets with due consideration on all the groups of users including children, older people, disable people, cyclists, young people, and families etc., without excluding any category of the society. As such, "inclusive design" can be considered as one of the main contributory factor for designing a successful SSS.

\subsection{Location and connectivity}

Department for Transport (2011), emphasize that the connectivity with the public transportation network is an important factor for a successful SSS design. However, this does not mean that the buses or trams should use the shared street as part of the route, but to be a pedestrian 
friendly street and to facilitate the reduction the vehicular movements, the street can be well-connected with the public transportation system.

Providing alternative routes to transfer the vehicular traffic is another way of ensuring connectivity within the context of SSS (Department for Transport, 2011). This is a requirement to maintain one of the key characteristics of SSS; the pedestrian prominence with low speed of traffic. Supporting this, Kaparias et al. (2013) state that redesigning urban street into shared spaces generally decrease the pedestrian-vehicle traffic conflicts. In order to achieve this Pharoah (2008) state that, the presence of alternative routes for vehicles is one of the main elements to maintain the smooth flow of traffic, as the street itself is designed for slow speed of traffic.

In summary, four main principles of Shared Space Streets were identified through the literature analysis which can be considered as four main objectives of a successful Shared Space Street; Pedestrian prominence, Distinctive and Attractive Public Place, Inclusive design and Location and connectivity. A set characteristics and contributory design factors have also been identified under each objective. However, considering the recent criticisms received on SSS, it is questionable whether the above identified factors in the literature are sufficient to achieve successful SSS design in general, or are there any other contributory factors that should be considered at operational level. Therefore, while the above literature findings were used as a theoretical base, an empirical study was conducted to achieve the aim of this research. In addition, the literature informed theoretical base provided the foundation for the field data to be collected in a systematic way for the empirical investigations.

\section{Research method}

To answer the central research question of this study a case study method was used for the empirical investigations. This method allowed the researchers to find the answer through an in-depth investigation within its real life, contemporary context. The theoretical proposition (leading to the unit of analysis) of this case study design was that "the concept of SSS can be successfully applied through the careful application of design interventions", because only the unpredictability of design and unfamiliarity with the concept, creates most of the issues (CABE, 2008). This study employed multiple cases as its case study strategy, as it facilitates theoretical generalisation and al- lows to examine several cases to understand the similarities and differences between shared space cases.

The case studies selection was done in two parts. Firstly, in order to maintain the consistency between the cases, the case streets were chosen within urban or urban fringe areas and only the commercial streets were taken without taking residential streets or other types of street into consideration. The main reason for this is the comparison between completely different categories of streets (rural or residential) does not permit the intended theoretical generalisation. Accordingly, three cases within United Kingdom (details provided below) were selected. Secondly, secondary data were collected under each case to assess whether these places have experienced the conventional street experience, problems with it and to find out the changes made through the conversion to SSS.

The data collection technique was observation which allowed researchers to understand how the design elements effect the street user's behaviour and to understand which elements worked well, and the reasons behind. This method supports the notion of Jacobs (1995), where he notes observation of examples as the best method to use for better design of public streets.

Furthermore, all the cases were assessed on daytime within a weekday. Also sufficient attentions were given on selecting a daytime on a weekday for all the cases without being impacted by any special events in a bid to eliminate any distortion to the collected observational data.

\section{Context of selected cases}

\subsection{Exhibition road London}

Exhibition road is located in South Kensington, London, UK and the main characteristic of this street is that, it provides access to many public places such as Natural History Museum, Victoria \& Albert Museum, Science Museum, Imperial College London, Hyde park, Royal Geographical Society and The Church of Jesus Christ of Latter-day Saints meetinghouse. According to the literature, this was previously a vehicle dominant road and was very inconvenient to the pedestrians including visitors, students, local workers and residents (The Royal Borough of South Kensington and Chelsea, 2012). Therefore, to make this as a pedestrian friendly street local authority of Royal Borough of Kensington and Chelsea placed as redesign project to convert it as a SSS (Figure 1).
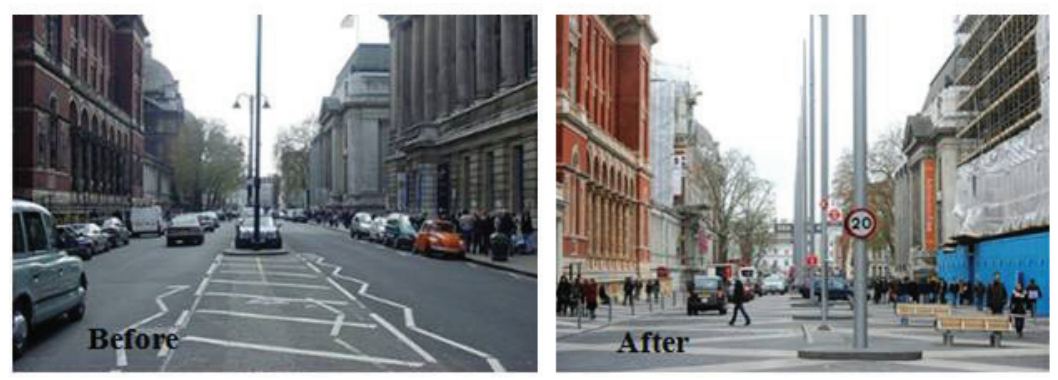

Figure 1. Changes made to exhibition road (www.rbkc.gov.uk) 


\subsection{Park Lane, Poynton, Stockport, UK}

Park Lane is located in the Poynton Town Center, Stockport which is the main shopping street to the local residents (Figure 2). Hamilton-Baillie (2007) notes that the street provides access to both divers who pass the area and pedestrian who come to visit retail shops and various service premises including saloons, solicitors, estate agent, travel agent, dentist, pharmacy and alike. The main characteristic of this street is the heavy traffic flow through the lane which marks some days as 10,500 per 12 hours. Hamilton-Baillie (2007) also reveals that the main reason for the conversion is the loss of continuity of pedestrian activities due to the vehicular traffic and resulted issues related to safety and anti-social behavior.

\subsection{Byng Place, London, UK}

The Byng Place located between the Torrington Place and the Gordon square in Bloomsbury, near to the University of London. The road is mainly used by visitors of the British museum, university students and the office workers in Bloomsbury. According to the project report, the main reason for the conversion of the Byng place as SSS is to "break the traffic" and enhance the visual partnership connecting the University Church and Senate House (Figure 3) (Farrell, 2006).

\section{Findings and analysis}

As it has been discussed, the conceptual framework was used as a base for the data collection. However, the central consideration of data collection was given on discovering all the potential design factors which can contribute in achieving the four main objectives. The main purpose of this central consideration was to keep it open for the new factors to be emerged without evaluating only the design factors on the framework. Accordingly, the case study findings will be discussed under the four main objectives of successful shared space street.

\subsection{Pedestrian prominance}

There is one level of surface in both Exhibition road and Park Lane which was observed as one of the main reasons for the free pedestrian movement. Adding to this, Byng place has a 1-2 ft. height gap between the carriage way and the pedestrian way and therefore, pedestrian always took the pedestrian way without crossing the boundaries. Accordingly, it was observed that, even though it is comparably a small gap, this separation works as a mental barrier separating the vehicular movements from pedestrian movements, somewhat limiting the pedestrian prominence.

In terms of maintaining a de-cluttered environment, all the three cases give the same message as the traffic signals and other signage were removed as much as possible in each case. All three cases maintain minimum number of traffic and other signs. For instance, Exhibition Road has residents permit holders' parking signs, blue badge holders' signs and signs for the London Underground. Park lane had only two types of signs; "do not drink" signs and the "bus stop" sign and Byng place also retains few including "parking restricted zone" sign and the "cycle and pedestrian way" sign. However, the unique characteristic of Exhibition Street is that, bollards have been used to divide the pedestrian way and carriage way, only at one section of the street where the traffic flow is high. Further, the
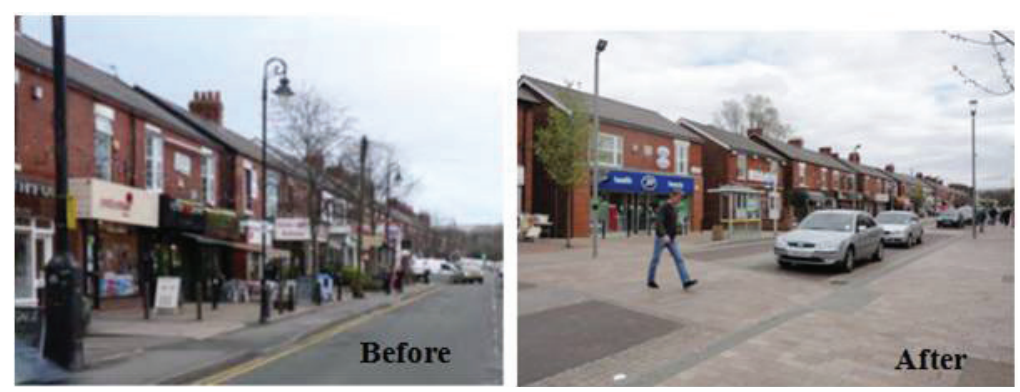

Figure 2. Changes made to Park Lane (www.hamilton-baillie.co.uk)
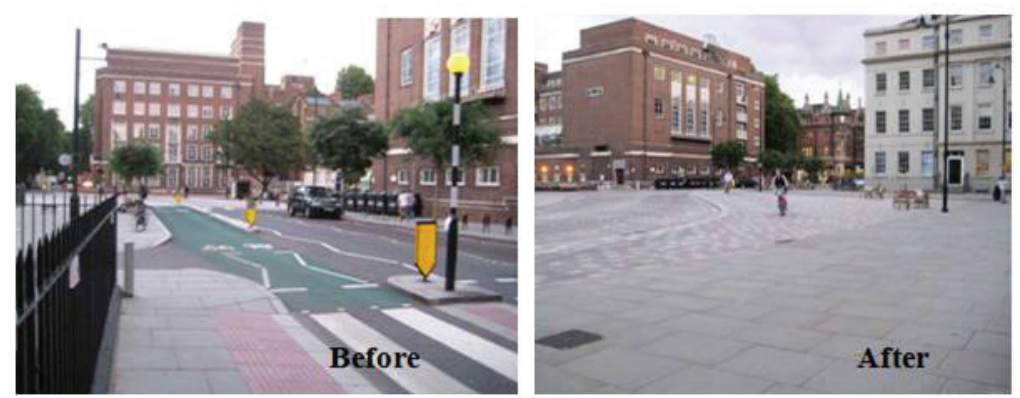

Figure 3. Changes made to Byng Place, London (www.voleospeed.blogspot.co.uk) 
other parts of the street were completely designed without traffic rules such as signals, signs, divisions, median lines, markings etc. Even the street furniture is arranged with a minimal disruption to the pedestrian activities, e.g. garbage collection bins are fixed to the lightning posts to create minimal disruption for the free movement of pedestrian. Accordingly, the observations highlighted the fact that successful SSS conversion does not mean the complete removal of all the traffic signs and other elements of conventional street. Some street elements can be left in order to maintain the safety and viability of the street, yet those elements need to be arranged maintaining the key objective of the street.

Another main factor which effects for the low speed of traffic and freedom of pedestrian movement is visually narrowed carriageway. The significance of this design element was present in all the three cases but in different ways. Especially, the Park Lane which is the main traffic distribution road of Poynton town centre, has maintained the low traffic speed through the visual narrowing. The main method of visual narrowing is that the narrowed carriage way which is defined through differentiating the colour of the paving material (Figure 4). Instead, one section of the Exhibition Street has used features such as the arrangement of parking spaces, street lighting, trees and drainage to narrow the visual space for vehicular movement. Further, Byng place has not used any type of visual narrowing which can be considered as the main reason for the speedy movement of traffic. Accordingly, it can be noted that, input of design elements for visual narrowing is one of the important features to slow down the traffic. In contrast, the entry section of the exhibition street does not use any type of design element for visual narrowing. Yet, it can be observed that the slow speed of vehicular movement is present there. The main reason for this is that the space is always congested with the pedestrians who visit the restaurants and cafeterias on both sides of the street. This emphasize that the visual narrowing of carriage way is a necessity and it could be achieved through the design of the space with distinctive paving colour or using street furniture, existence of pedestrian and activities, or maybe mixture of these elements.

Allocation of parking slots is also confirmed by the case study analysis as a design factor promoting pedestrian prominance. Exhibition Road is mainly a parking restricted zone and limited parking bays have been provided for residents and blue badge holders. More importantly, these parking areas were placed between the pedestrian safe area and carriage way which also act as a transitional zone (approximately $8 \mathrm{~m}$ wide area) for pedestrian activities (Figure 5). Furthermore, street furniture such as benches and cycle racks are placed between pedestrian areas and parking areas in order to protect the pedestrian safe area from interference of parking activities.

In Park Lane, all parking spaces are allocated separately at the rear of each building plot. The main reason for this type of allocation may be the width of Park Lane which does not allow the design of on street parking. However this resulted in a pedestrian friendly environment within the street. Further, parking is restricted in Byng Place due to size of the area and there are parking spaces available for surrounding land uses.

The above observations highlight that, for the successful application of SSS, the allocation of parking spaces for
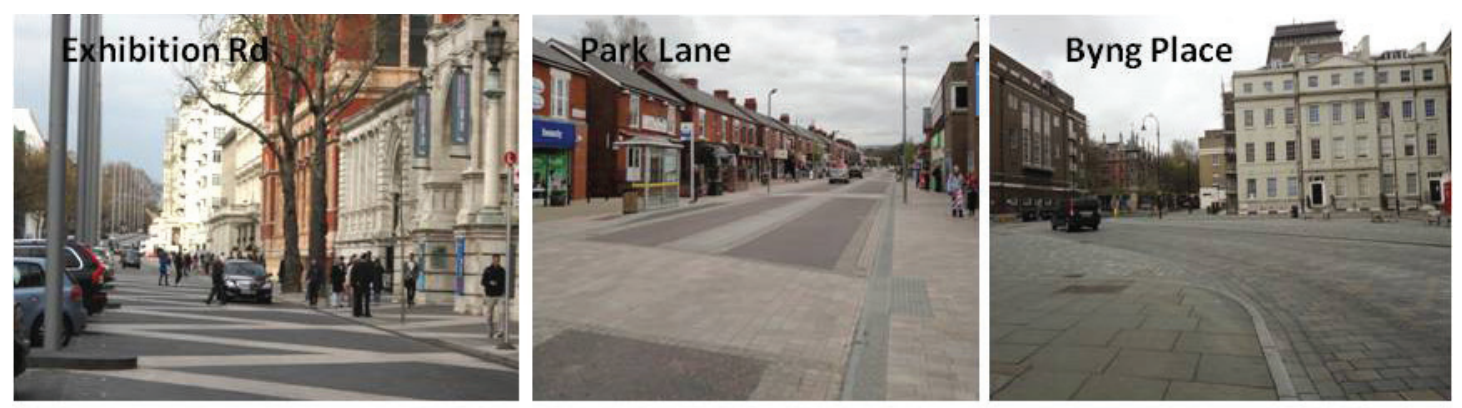

Figure 4. Visual narrowing in case studies

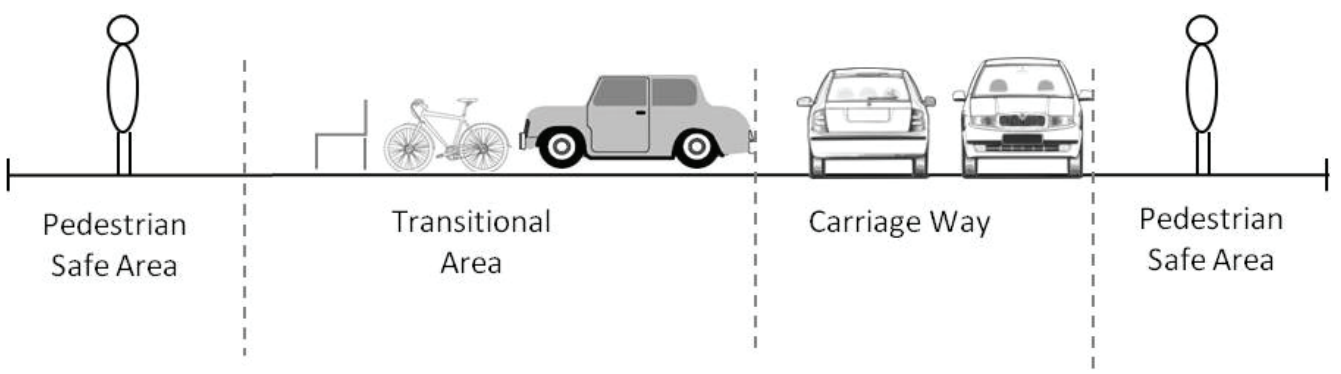

Figure 5. Cross section of Exhibition Road 
vehicles and cycles should be considered without interrupting the pedestrian activities. The pattern of allocation may vary according to the setting as in Park lane and Byng Place. However, if the parking is allowed on street it may be designed without any interruption to pedestrian activities as seen in the Exhibition Road.

Apart from the above four factors which were confirmed by the case study findings, another two factors were immerged from the case study findings giving slightly different perspectives to the literature review findings. The surface design of Exhibition Street is the most unique feature of this shared street which creates the feeling of "one surface". Especially, the chequered surface design throughout the road surface with thick white strips marked angularly from one corner to the other gives the impression to the pedestrians that the "entire space is yours", "you can move wherever desired direction". This message has strongly immerged from the observations of how the pedestrian move and how they use the space. In contrast, Poynton Street is visually separated between carriage way and pedestrian way in support of traffic safety. However, based on the observed behaviour pattern of the pedestrians, it is identifiable that it is still convenient for pedestrians to cross the street using courtesy crossings and they use it freely. The underlying reason observed for this is the provision of courtesy crossings which deliver number of desired lines to cross the street and the texture of crossing is same as the foot way which gives the feeling to treat crossings as part of the foot way. Accordingly, it has been observed that the surface design has a great potential to contribute to the pedestrian prominence through maintaining the characteristics of the sense of one single space.

Second immerged factor is the entrance design or the gateway design. The entrance to Park Lane is designed with wide stone paved roundabouts in both ends which are not controlled by traffic signals. It effectively slows down vehicles. In contrast, Exhibition road or Byng place do not feature a gate way design, yet, the unique surface design of the exhibition street gives the feeling to the vehicles that they are entering into a different zone and existence of pedestrian and other design elements maintain the slow traffic flow throughout the street. However, in Byng place the stone paving gives the feeling of entering to a different zone, but due to the fact that there is no visual narrowing and no pedestrian activities, vehicles tend to move faster as in a conventional street.

In addition to the above, the case study analysis introduced a new factor for better SSS design; pedestrian safe way. In the Exhibition Street, separate area is allocated for pedestrian use without any interference from vehicles and cycles which also provides access to the main public attractions within the street; the Natural History Museum and the Science Museum (Figure 5). Park Lane also introduces the idea of having pedestrian safe area, but with two sections on it; pedestrian area (Section 'A' of Figure 6), and pedestrian + shop area (Section 'B' of Figure 6).

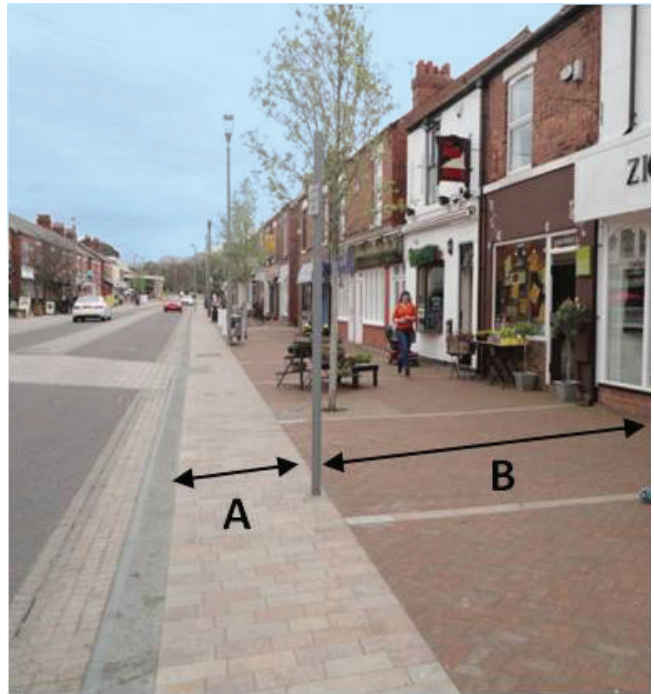

Figure 6. Pedestrian safe area in Park Lane

This feature allows pedestrians a choice whereby people who wants to move without any interruption can select the pedestrian way and for those who wish to visit shops can use pedestrian and shop way which includes café seating facilities, shop signage, commodity displays etc. The main feature of section $B$ is that it acts as a semipublic area contributing to function the place as an attractive place. Further, Byng place also has a separate foot way (Figure 7), but the layout of the "L" shaped footway discourages pedestrians from using it and instead to use the pedestrian and cycle path. The main reason for this is that, the nature of human beings is such that they tend to take the shortest path between two points if they use the place just for the purpose of movement.

Based on the above, consideration of a pedestrian safe pathways can be identified as an important feature for the success of a SSS, for the reason that this factor effectively answers the main criticism of this concept which is "Design not for all". This means, if the demarcated pedestrian pathways are not provided, as in a conventional street, older people or people with hearing needs may find difficulties due to being unable see or hear vehicle movement. Therefore, incorporating this feature into a SSS, people with special needs can use the pedestrian safe zone be-

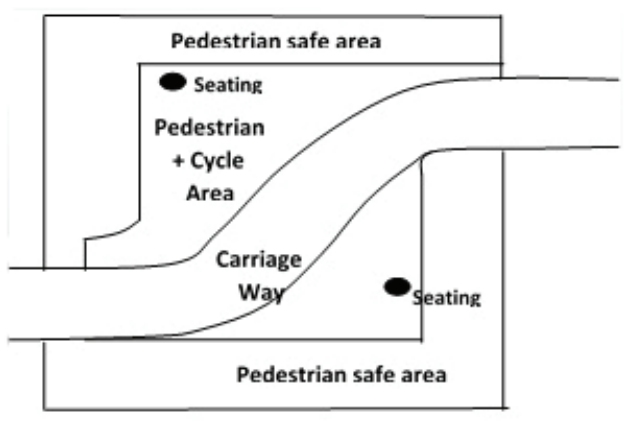

Figure 7. Layout of Byng place 
cause there is no interruption from vehicles or cycles. At the same time, based on the observations from the case studies, at the design stage particular consideration can be given to the functionality of the street and the connectivity of the pedestrian safe way with the adjoining layers of the street and other activities.

In summary, the objective of pedestrian prominence can be achieved through maintaining three main characteristics which are; convenience in pedestrian movement, low traffic speed and sense of a single space. Further, the case study analysis established seven design factors that can be used to maintain the identified three characteristics. Those seven design factors are; level of surface, surface design, de-cluttered environment, allocation of parking space without interrupting pedestrian activities, allocation of pedestrian safe area, visually narrowed carriage way and gateway design.

\subsection{Distinctive and attractive public place}

Literature analysis identified the use of active frontage of building promoting the idea of availability of publicly accessible ground floor use and the provision of doors and windows facing to the street as design features for distinctive and attractive public places. The need of this character was specially confirmed at the Park lane and one section of the Exhibition street. Park lane maintains active frontage throughout the street which contributes to increase the public footfall for shopping, for other service uses and in some cases, just window shopping. Further, the Exhibition street al.o uses the active frontage at the entrance section where the main attractive public place within the street with shops, cafés and restaurants are placed. However, the active frontage character is missing at the main section of the street with museums and institutions. Nevertheless, the street is still distinctive and attractive because of the existence and the activities of the visitors who come to the main attractions. Conversely, the Byng place also surrounded by a church, park and institution, yet does not act as distinctive and attractive to the public due to the isolation of the location and for not maintaining the active frontage. Accordingly, within the case studies it has been observed that the active frontage of buildings is an important factor for the attractiveness and the distinctiveness. However, if the surrounding area features public at- tractions and increases the public foot fall across the area, active frontage may not be a necessity. Accordingly, the surrounding and the vicinity which attract public to the street also one of the main drivers to achieve distinctive and attractive public place within the SSS and this is a new factor, identified through case study analysis, which was not prominent in the literature informed conceptual framework.

The second factor which was identified by the literature analysis and was also confirmed by the case study analysis is the allocation of comfort places. This character can be seen in Park lane which function well as most of the shoppers use this place as a resting place due to the presence of seating facilities. In Exhibition road, there is no special allocation of comfort place. However, the places in pedestrian safe zone where the seating facilities are provided, act as comfort places. Further, Byng place also has included seating facilities within the pedestrian and cycle area (Figure 8), but not within the pedestrian safe zone. It is observed that, street users do not use this place as a comfort place as the seating facilities are provided at the centre of a wide space without any "sense of enclosure".

The case study observations confirmed that the, allocation of comfort places can be considered as an important factor to achieve the objective of providing a place to rest and promote various pedestrian activities. However, the case study analysis emphasized the importance of the provision of seating facilities as an essential element for comfort places ensuring such allocation matches the behaviour patterns of people.

Apart from that, designing for outdoor public and private activities have a significant potential to contribute to the above objective and this was confirmed within both literature review and case study analysis. For instance, Park lane maintains a semi-public area between the shops and pedestrian way which promotes people to stay for a while, function as an outdoor café area, to display the billboards and advertisement boards. This allocation add value to function as a distinctive and attractive place.

Fourth factor observed was the visual quality of place. This emphasize the design considerations specifically on the visual quality of lighting, street elements and surfaces. One of the main features of Exhibition street is the lighting column which runs throughout the street at the

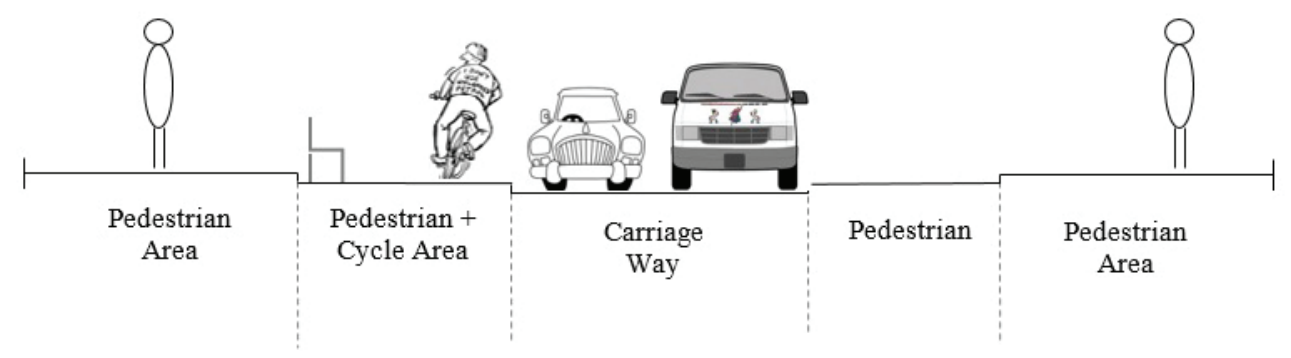

Figure 8. Cross section of Byng place, London 
middle with a height of approximately $20 \mathrm{~m}$. This feature provides uniqueness to the road on the day time plus a distinct feature and covers the safety element at night time. Apart from that, the colors of the street furniture including lighting columns, benches and bins are in grey or silver color matching the street surface with grey and white which also provides the visual quality to the place. However, Byng place street furniture; benches, wooden chairs and tables do not add notable visual quality to the place and not even the colors of the paving. This feature added with the other negative features (e.g. not having active frontage) prompted this not to function as distinctive and attractive public place as intended.

Apart from the main factors which were identified through the literature review, the case study analysis revealed the importance of new factor; visual linkage with adjoining public and semi-public places. This factor was identified especially in the case of Byng place. This shared space is located at the middle of Toronto Square and the Gordon Square Garden (Figure 9), which means the area is physically linked with a public park and open spaces. However, the Byng place do not have any visual linkage with both places which is a disadvantage for the shared street to function well as a distinctive and attractive place. Due to this reason the people who use the Gordon square garden do not tend to use this space as an attractive place. In contrast, Exhibition road and Park lane both had the visual linkage with surrounding public and semi-public places as both were straight streets.

In summary, the objective of having distinctive and attractive public places can be achieved through the maintenance of design of activities to attract to the place, visual attractiveness and active engagement of people with the space. Further, the case study analysis introduced six design factors to maintain the above characteristics; active frontage; linkage with main public attractions; design for outdoor public and private activities; allocation of comfort places; visual quality of space and visual linkage with other public and semi-public places.

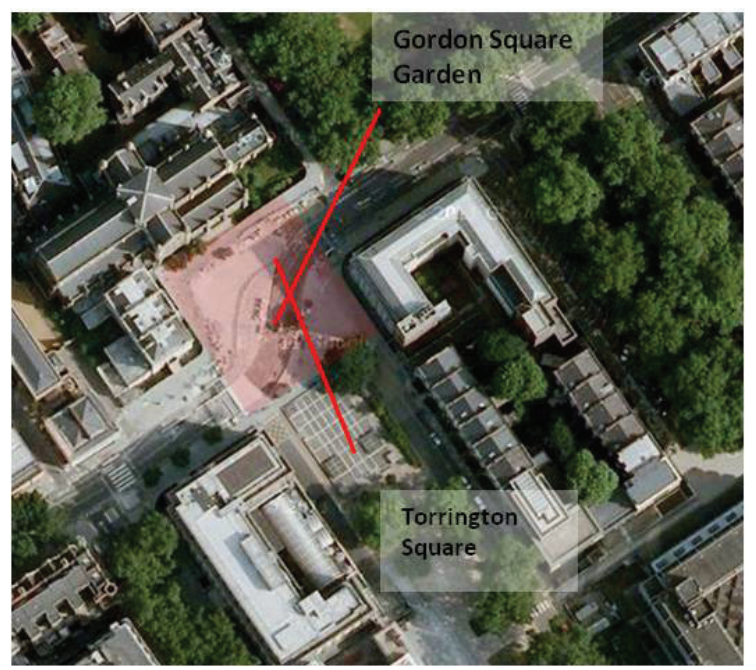

Figure 9. Possible visual linkage at Byng place

\subsection{Inclusive design}

The objective of inclusive design was identified as a measure to counter the main criticism of SSS, which is the difficulties encountered by older people and people with special needs. Even though the need was identified through the literature, specific set of design factors could not be identified to achieve this objective due to the lack of literature covering this aspect in relation to SSS. However, the case study analysis discovered five main design factors related to this. Though, most of the factors identified in this section overlap with factors identified elsewhere in the paper, due consideration has been given from the inclusive design perspective.

Case study analysis reveals that levelled surface of SSS can benefit for people with walking difficulties especially for wheelchair users. They can move any direction within the street as there is no level differentiation to divide carriage way and cycle lane. However, the main criticism is that the level of surface is dangerous for blind or partially sighted people and for people with hearing difficulties. Based on the observations, the corduroy/tactile paving which divides the carriage way and pedestrian way, assist the people with these type of difficulties to identify the pedestrian way. Conversely, in Park lane, a different stone paving with a grip is used to separate the carriage way from the pedestrian way where even blind or partially sighted people can feel when they walk and people with hearing difficulties can see the different coloured paving.

Though theses design strategies were installed to benefit the people with special needs, the argument is still valid that these elements cannot eliminate the "feeling of being unsafe" to be on this type of street. However, allocation of pedestrian safe way which was identified at the pedestrian prominence section can answer to this criticism. As discussed, a pedestrian safe zone which is protected from the carriage way using a transitional zone with parking lots, cycle racks and benches (Figure 5), can be used for people with special needs. Confirming this, in the Exhibition street most of the people with special needs chose the pedestrian safe zone for movement.

Seating facilities is another factor which can contribute to inclusive design. From the perspective of inclusive design, the shared space should be designed with seating facilities with a regular frequency. Comparing the two case studies Exhibition road and Park lane, the Exhibition street has provided a considerable amount of seating facilities at regular distances, whereas in Park lane there is only one public seating area at the entrance of the street. Due to this reason, it was observed that the street users, especially the aged people had to come all the way back to entrance point if they want to take a rest. Hence it is noted that, seating areas are needed to be provided within the comfort zone to give the feeling of safety and comfort and also important consideration need to be given on the availability of seating facilities at regular distances.

While a suitable balance should be maintained between the provision of parking facilities and disturbance 
to pedestrian activities, parking provision for Blue Badge Holders still need to be considered appropriately. The Exhibition street provides a good case example for a solution on provision of street parking for blue badge holders without disturbing the footway. The fifth design factor is the design for diverse activities for different age groups where the consideration can be given for children and young people. For instance, in Park lane group of young people were playing roller skate using the steps at the entrance of a shop. If provision were made within the street layout this would be an added functionality for the street. Further, in the Byng place case, people did not have any reason to stay in this place other than moving. Therefore, if provisions were made for aspects such as design for children to play it would benefit to achieve both objectives; inclusive design and distinctive and attractive place.

In summary, the objective of inclusive design can be achieved through three main characteristics; ease of movement, sense of safety and welcoming for all. Further, these characteristics can be achieved through five design factors; Level of surface and surface material, pedestrian safe way, regular seating facilities and design for diverse activities for different age groups.

\subsection{Location and connectivity}

In terms of the connectivity with public transportation network, Exhibition Street is connected with the public bus routes through the Cromwell road which is one of main bus routes in London. Further the street is connected with two main underground stations which provide the access to majority of the visitors to the place. The Park lane is also located at a main junction and linked with local bus routes and so as the Byng place. Based on the analysis of the case studies this well-established connectivity with the public transportation network as well as with the public attractions has a great impact to the functionality of the street.

Further, the importance of the connectivity with alternative routes to transfer the traffic was identified at the Exhibition road case study where there are number of alternative routes to bypass the pedestrian traffic and move freely. However, in Park lane there were no alternative routes for vehicles to move freely and therefore, on peek-hours this caused problems to both vehicles and pedestrians. Accordingly, it is well observed that the availability of alternative routes acts as an added advantage for the successful application of a SSS.

Apart from these factors, the case study analysis presented a new factor under this category which is the "the need for a shared space". The two main characteristics of a shared street are pedestrian prominence and allowing all three users; pedestrian, vehicles and cycles to use the street. In other words, for the conversion from conventional street to shared street, there should be a need of pedestrian prominence and sharing the street by all type of transportation. "Need for a Shared Space" at Exhibition Road, was emerged as the main users of the street are pe- destrians; including tourist visiting the Science Museum, university students and visitors to other institutions. Further, literature confirmed that there was a need for street conversion as the dominance of the wide carriage way was inconvenient for pedestrians including visitors, students, local workers and residents (The Royal Borough of South Kensington and Chelsea, 2012). Accordingly, the "Need for a Shared Space Street" was emerged. Similarly, Park Lane is the main shopping area which caters to the surrounding residential area and the street is mostly used by shoppers of the local area, pedestrian flow should be the main focus of the street. Yet, the street should be open to vehicles and cycles as it gives main access to the local area. However, in case of Byng place, the pedestrian prominence is not needed as the place is not attracted by many pedestrians and neither gives access to public attractions. Accordingly, it can be understood that, if there is no need of converting to shared space the conversion will not be successful even though all the other design factors are implemented. Further, it will create additional issues to the place rather than solving an existing issue.

\section{Conclusion}

The paper established the importance of achieving four main objectives for the successful application of SSS concept. Further, the paper developed and presented a set of characteristics to be maintained under each objective and set of design factors so that the four objectives are achieved through the maintenance of these characteristics.

Accordingly, the objective of pedestrian prominence can be achieved through maintaining three main characteristics; convenience in pedestrian movement, low traffic speed and sense of a single space. These three characteristics can be maintained through the application of seven design factors: level of surface, surface design, de-cluttered environment, allocation of parking space without interrupting pedestrian activities, allocation of pedestrian safe area, visually narrowed carriage way and gateway design. Secondly, the objective of distinctive and attractive public place can be achieved through the maintenance of three characteristics; visual attractiveness, activities to attract to the place and active engagement of people with the space. Further, these characteristics can be maintained through the application of six design factors; active frontage, linkage with main public attractions, design for outdoor public and private activities, allocation of comfort places, visual quality of space and visual linkage with other public and semi-public places. Thirdly, the objective of inclusive design can be achieved through maintaining three main characteristics; ease of movement, sense of safety and welcoming for all. Further, these characteristics can be achieved through five design factors; level of surface and surface material, pedestrian safe way, seating facilities with regular frequency and design for diverse activities for different age groups. Fourth objective which is the location and connectivity can be achieved through the connectivity with public transportation network, connectivity with 


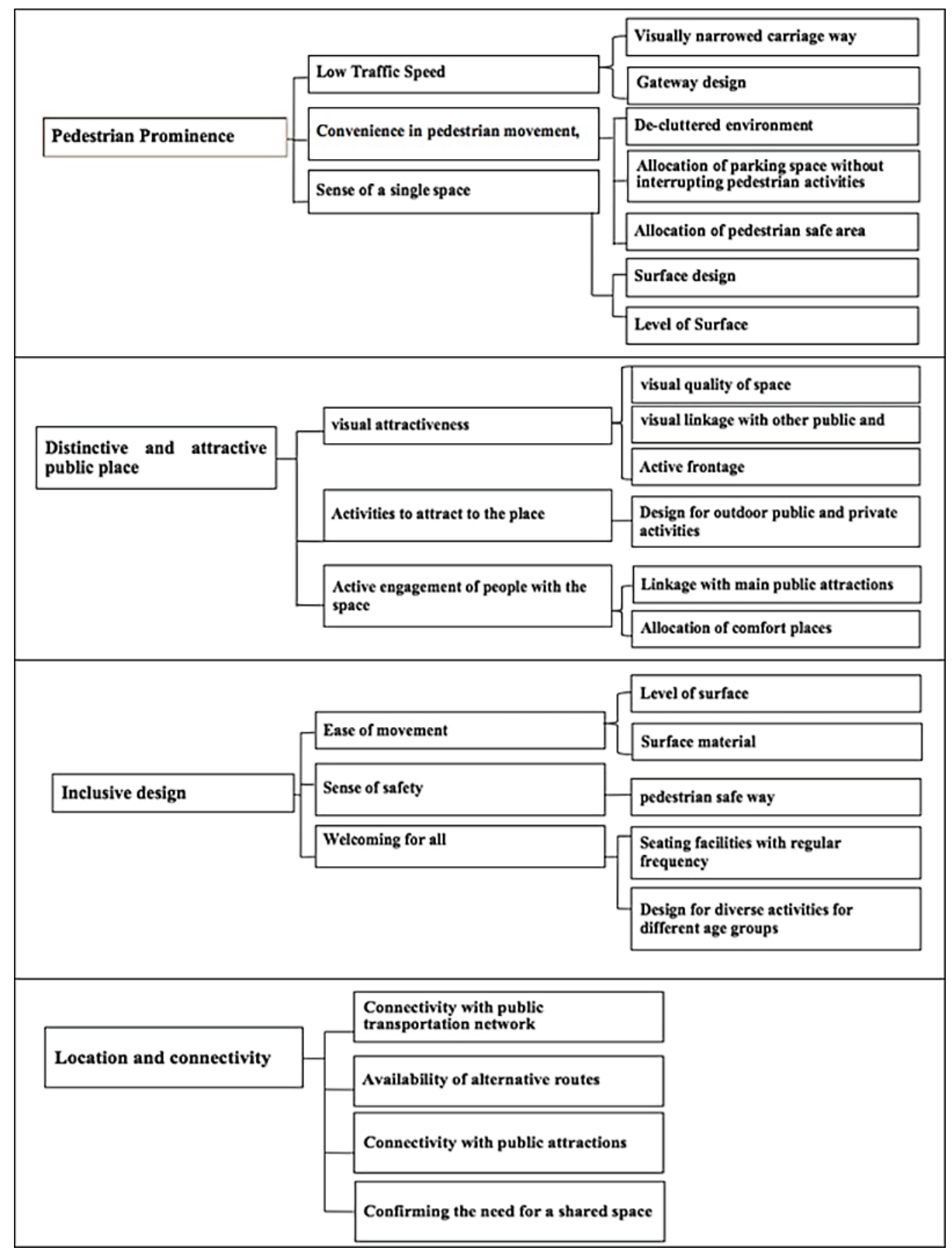

Figure 10. Design factors for successful shared space street

public attractions, availability of alternative routes and confirming the need for a shared street.

Finally, based on the above discussion, a framework for the successful application of shared spaces street can be visually represented as follows (Figure 10). Some of the elements of this framework may have a more significant contribution for the successful application of SSS than the other elements which was not evaluated at this research study. Therefore, future researchers may wish to evaluate the level of significance of these characteristics which is beyond the scope of this paper. Findings of this research can be used by the urban design and planning practitioners as a framework for the successful application of the concept along with the consideration of context specific factors.

\section{References}

Babbage Science and technology. (2012). Crowd dynamics: walk this way. Retrieved from http://www.economist.com/node/21544803

Burton, E., \& Mitchell, L. (2006). Inclusive urban design: streets for life. Oxon: Routledge.

CABE. (2008). Commission for Architecture and the Built Environment $(C A B E)$. Retrieved from http://webarchive.nationalarchives.gov.uk/20110118095356/http:/www.cabe.org.uk

Carmona, M., Tiesdell, S., Heath, T., \& Oc, T. (2003). Public places urban spaces ( $2^{\text {nd }}$ ed.). Burlington: Elsevier Publications.

Curl, A., Thompson, C. W., \& Aspinall, P. (2015). The effectiveness of "shared space" residential street interventions on selfreported activity levels and quality of life for older people. Landscape and Urban Planning, 139, 117-125. https://doi.org/10.1016/j.landurbplan.2015.02.019 
Department for Transport. (2011). Local transport note 1/11: shared space street. Retrieved from http://assets.dft.gov.uk/ publications/ltn-01-11/ltn-1-11-quantitative.pdf

Farrell, T. (2006). Bloomsbury, a strategic vision. Retrieved from http://www.camden.gov.uk/ccm/cms-service/stream/ asset/?asset_id=2078939

Gehl, J. (2013). Cities for people. Island press.

Gerlach, J., Methorst, R., Boenke, D., \& Leven, J. (2008). Sense and nonsense about shared space. Retrieved from http:// www.fietsberaad.nl/library/repository/bestanden/Shared\%20 Space_short_german-Eng.pdf

Hamilton-Baillie Associates. (2007). CREWKERNE a fresh approach to streets, traffic and public spaces. Retrieved from http://www.somersettownsforum.org.uk/uploads/documents/ crewkernestreetsparkingreport.pdf

Hamilton-Baillie, B. (2008). Shared space: reconciling people, places and traffic. Built Environment, 34(2), 161-181. https:// doi.org/10.2148/benv.34.2.161

Healthy Cities. (2012). Designing streets for different users - New Road, Brighton and Hove. Retrieved from http://healthycities. org.uk/uploads/files/010_designing_street_for_different_users__brighton_and_hove.pdf

Hebbert, M. (2003). New urbanism - the movement in context. Built Environment, 29(3), 193-209. https://doi.org/10.2148/ benv.29.3.193.54285

Holmes. L. C. (2015). Accidents by design: the Holmes report on "shared space" in the United Kingdom. Retrieved from https:// www.theihe.org/wp-content/uploads/2013/08/Holmes-Report-on-Shared-Space-.pdf

Imrie, R. (2012). Auto-disabilities: the case of shared space environments. Environment and Planning A, 44(9), 2260-2277. https://doi.org/10.1068/a44595

Jacobs, A. B. (1995). Great streets. Cambridge, MA: MIT Press.
Kaparias, I., Bell, M., Dong, W., Sastrawinata, A., Singh, A., Wang, X., \& Mount, B. (2013). Analysis of pedestrian-vehicle traffic conflicts in street designs with elements of shared space. Transportation Research Record, 2393, 21-30. https://doi.org/10.3141/2393-03

Karndacharuk, A., Wilson, D. J., \& Dunn, R. (2014). A review of the evolution of shared (street) space concepts in urban environments. Transport Reviews, 34(2), 190-220. https://doi.org/10.1080/01441647.2014.893038

MacMichael, S. (2009). Oxford Circus gets shared space crossing as naked streets momentum grows. Retrieved from http://road. cc/content/news/10654-oxford-circus-gets-shared-spacecrossing-naked-streets-momentum-grows

Matthew, B. (2012). Country observer: news. Retrieved from http://www.coventryobserver.co.uk/2012/02/24/news-Petitions-to-axe-shared-space-junctions-ignored-31235.html

Pharoah, T. (2008, October). Shared space in action. The $9^{\text {th }}$ Walk21 International Walking Conference A Moving City. Barcelona, Spain.

Public Realm Information \& Advice Network. (2011). Mixed use streets: 5 characteristics of successful shared space. Retrieved from http://www.publicrealm.info/faq's/shared_space_case_ study.pdf

Talen, E. (1999). Sense of community and neighbourhood form: an assessment of the social doctrine of new urbanism. Urban Studies, 36(8), 1361-1379. https://doi.org/10.1080/0042098993033

The Guide Dogs for the Blind Association. (2014). Shared surfaces. Retrieved from http://www.guidedogs.org.uk/supportus/ campaigns/streets-ahead/shared-surfaces

The Royal Borough of South Kensington and Chelsea. (2012). The exhibition road. Retrieved from http://www.rbkc.gov.uk/ subsites/exhibitionroad.aspx 